\title{
Horizontal ducting of sound by curved nonlinear internal gravity waves in the continental shelf areas
}

\author{
Ying-Tsong Lin ${ }^{\mathrm{a})}$ \\ Applied Ocean Physics and Engineering Department, Woods Hole Oceanographic Institution, \\ Woods Hole, Massachusetts 02543 \\ Kara G. McMahon ${ }^{\text {b) }}$ \\ Mathematical Sciences Department, Rensselaer Polytechnic Institute, Troy, New York 12180 \\ James F. Lynch \\ Applied Ocean Physics and Engineering Department, Woods Hole Oceanographic Institution, \\ Woods Hole, Massachusetts 02543 \\ William L. Siegmann \\ Mathematical Sciences Department, Rensselaer Polytechnic Institute, Troy, New York 12180
}

(Received 2 July 2012; revised 6 November 2012; accepted 12 November 2012)

\begin{abstract}
The acoustic ducting effect by curved nonlinear gravity waves in shallow water is studied through idealized models in this paper. The internal wave ducts are three-dimensional, bounded vertically by the sea surface and bottom, and horizontally by aligned wavefronts. Both normal mode and parabolic equation methods are taken to analyze the ducted sound field. Two types of horizontal acoustic modes can be found in the curved internal wave duct. One is a whispering-gallery type formed by the sound energy trapped along the outer and concave boundary of the duct, and the other is a fully bouncing type due to continual reflections from boundaries in the duct. The ducting condition depends on both internal-wave and acoustic-source parameters, and a parametric study is conducted to derive a general pattern. The parabolic equation method provides full-field modeling of the sound field, so it includes other acoustic effects caused by internal waves, such as mode coupling/ scattering and horizontal Lloyd's mirror interference. Two examples are provided to present internal wave ducts with constant curvature and meandering wavefronts.
\end{abstract}

(C) 2013 Acoustical Society of America. [http://dx.doi.org/10.1121/1.4770240]

PACS number(s): 43.30.Bp, 43.20.Bi, 43.20.Mv [JAC]

Pages: $37-49$

\section{INTRODUCTION}

Nonlinear internal waves on the continental shelf and in shelfbreak regions can influence underwater sound propagation significantly. For sound propagating across the waves, the mode coupling, scattering, and resonant types of effects are most profound (Zhou et al., 1991; Preisig and Duda, 1997). Nonlinear internal waves can also act as reflective fronts, so horizontal Lloyd's mirror effects can occur (Badiey et al., 2011; McMahon et al., 2012). When two nonlinear internal waves align, a three-dimensional (3-D) acoustic duct may be formed which can potentially trap sound over a long distance (Katsnelson and Pereselkov, 2000; Oba and Finette, 2002; Finette and Oba, 2003; Badiey et al., 2005; Frank et al., 2005) and radiate sound from its open-ended termination (Lin et al., 2009). Most of the previous work on internal wave ducting concerns straight fronts and ducts, as they represent parts of the wave fields observed in (approximately) straightline continental shelfbreak regions such as the Mid-Atlantic Bight (Apel et al., 1997; Tang et al., 2007). However, curved internal wavefronts are just as common as linear fronts because

\footnotetext{
a) Author to whom correspondence should be addressed. Electronic mail: ytlin@whoi.edu

${ }^{b}$ Current address: MIT Lincoln Laboratory, Lexington, MA 02173.
}

wave generation from small (point-like) regions, diffraction by islands or seamounts, or refraction by bathymetry and/or oceanography can easily produce significant wavefront curvature. Theoretical analyses, numerical simulations, and observations of sound propagation in curved internal wave fields have been reported by Lynch et al. (2010), Duda et al. (2011), and McMahon et al. (2012). In this paper, a 3-D normal mode theory is employed to analyze the modal structure of the curved ducts. In addition, a 3-D parabolic equation method is used to calculate full sound field solutions and to demonstrate different ducting situations predicted from normal mode theory.

Nonlinear internal waves of depression can produce acoustic total internal reflection/refraction within a grazing angle about $5 \mathrm{deg}$ with respect to the wavefronts. Although the critical grazing angle seems small, 5 to $10 \mathrm{~dB}$ fluctuations in sound intensity can be expected and are observed (see the references heretofore). Curved internal wavefronts can in fact yield a sequence of sound reflections along its concave side and produce an oceanic whispering gallery effect, which may also be seen in the horizontal ducting of sound by a sound speed front over a slope (Lynch et al., 2006; Lin and Lynch, 2011). This whispering gallery effect has been long studied in structural acoustics, and the most famous example is the whispering gallery in St. Paul's Cathedral, first studied 
mathematically by Rayleigh (1910). This type of ducting phenomenon has also been found on solid elastic cylinders imbedded in a fluid (Frisk et al., 1975; Dickey et al., 1976) and many other instances over concave surfaces. The whispering gallery effect has also been exploited beyond acoustics, e.g., laser cavities in X-rays (Braud, 1992) and a recent work on optic resonators (Righini et al., 2011).

Lynch et al. (2010) presented theoretical analysis using geometric and ray acoustics for a variety of ducting conditions in curved internal wave fields. In this paper, 3-D normal mode and parabolic equation (PE) methods are employed. The modal analysis is carried out in a cylindrical coordinate system, where horizontal modes are cylinder functions. A numerical program implementing the uniform asymptotic expansions of Bessel functions (Abramowitz and Stegun, 1964; Temme, 1997) is utilized in the examples shown here. This numerical program was obtained from Dr. K. R. Hiremath of Zuse Institute Berlin, Germany, and it was used to calculate modes of optical waves propagating along bent slab waveguides (Hiremath et al., 2005), where whispering gallery modes are also observed.

This paper is organized as follows. In Sec. II, an idealized model of curved internal waves is presented, and the 3-D normal mode and PE methods are overviewed. The characteristics of curved internal wave acoustic ducts are analyzed with the normal mode method in Sec. III. Numerical examples are shown in Sec. IV, followed by a discussion in Sec. V. The paper is concluded in Sec. VI and directions for future work will be discussed.

\section{METHODS}

An idealized model for a curved internal wave duct is first proposed and described. Second, the two methods used in this paper to analyze the horizontal ducting of sound by curved internal waves are explained. The first method utilizes separation of variables to decompose the ducted sound field into vertical and horizontal modes. The second method employs a PE approximation to the 3-D Helmholtz wave equation and calculates the full sound field with a forward marching algorithm.

\section{A. Internal wave model}

An idealized model consisting of curved internal waves with circular fronts is employed, along with a two-layer water column model where the upper layer has higher sound speed. The thermocline in the water column is depressed by the internal waves as shown in Fig. 1(a). The radius of the circular front indicates its curvature, and the waves are separated by a constant distance. To simplify the theoretical analysis, the internal waves are assumed to be a square waveform, which is the first order stair-step approximation to realistic internal solitary waves (Preisig and Duda, 1997; Lin et al., 2009).

With a cylindrical coordinate system $(r, \theta, z)$ as shown in Fig. 1(b), the 3-D sound pressure field $P(r, \theta, z)$ between the internal waves in both the water column and the bottom is governed by the following Helmholtz equation:

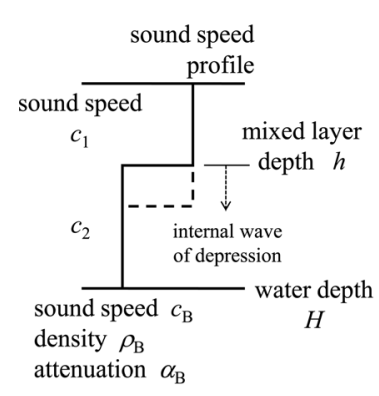

(a)

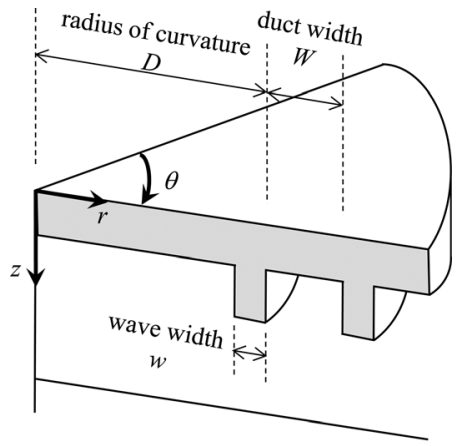

(b)
FIG. 1. An idealized model of a curved internal wave duct. (a) A two-layer water column perturbed by internal waves of depression. (b) Side view of an internal wave duct using the water column in (a). The shaded area indicates the upper layer with sound speed $c_{1}$ higher than sound speed $c_{2}$ in the lower water column.

$$
\frac{1}{r} \frac{\partial}{\partial r}\left(r \frac{\partial P}{\partial r}\right)+\frac{1}{r^{2}} \frac{\partial^{2} P}{\partial \theta^{2}}+\rho(z) \frac{\partial}{\partial z}\left(\frac{1}{\rho(z)} \frac{\partial P}{\partial z}\right)+\frac{\omega^{2}}{c^{2}(r, z)} P=0,
$$

where $c(r, z)$ is the medium sound speed without considering azimuthal variability, $\rho(z)$ is the medium density neglecting horizontal variation, and $\omega=2 \pi f$ is the angular frequency of the sound waves. The sound speed $c$ in the bottom is complex because the medium absorption is included, and the attenuation coefficient appears in the imaginary part.

\section{B. Acoustic mode decomposition}

Due to the presence of the sea surface and the sea floor, the long-distance propagating sound is guided in the water column with vertical acoustic modes. The vertical mode $\psi_{m}(z)$, where the subscript $m$ indicates the mode number, varies with horizontal position and can be determined from the local mode equation

$$
\rho(z) \frac{d}{d z}\left(\frac{1}{\rho(z)} \frac{d \psi_{m}}{d z}\right)+\left(k^{2}(r, z)-\zeta_{m}^{2}\right) \psi_{m}=0,
$$

where $k=\omega / c$ is the medium wavenumber and $\zeta_{m}$ is the eigenvalue of the $m$ th vertical mode, i.e., its horizontal wavenumber determined from the boundary conditions at the sea surface and bottom. Since the medium wavenumber $k$ is a function of $r$, the vertical mode $\psi_{m}$ and its horizontal wavenumber $\zeta_{m}$ can vary in $r$. Solving for the vertical modes is a well-established subject, and the details are omitted here. Readers are referred to Pekeris (1948) and Jensen et al. (1994) for further discussion. Here we will focus on the horizontal modes.

Because we are interested in ducted sound propagating along the circular fronts in the angular direction, we will neglect vertical mode coupling across the internal waves in analysis of the horizontal modes. This is the so-called adiabatic mode assumption and has been shown to be effective for analyzing the horizontal reflection and refraction of sound within a small grazing angle (less than $10 \mathrm{deg}$ ) with respect to frontal interfaces (Katsnelson and Pereselkov, 2000; Lin et al. 2009; Badiey et al., 2011). 
The depth averaged sound speed in the water column at wave locations is higher compared with places without waves because the internal waves induce depression of the thermocline. Thus, there is a potential for sound to be trapped in the duct between the waves. To find the horizontal modes of ducted sound, one can substitute the vertical mode decomposition $P(r, \theta, z)=\sum_{m} A_{m}(r, \theta) \psi_{m}(z)$ for the pressure in Eq. (1) and apply the adiabatic mode assumption. This will result in the following horizontal refraction equation for $A_{m}$,

$$
\frac{1}{r} \frac{\partial}{\partial r}\left(r \frac{\partial A_{m}}{\partial r}\right)+\frac{1}{r^{2}} \frac{\partial^{2} A_{m}}{\partial \theta^{2}}+\zeta_{m}^{2} A_{m}=0 .
$$

Employing the method of separation of variables, $A_{m}(r, \theta)=\sum_{n} G_{m n}(r) F_{m n}(\theta)$, we can rewrite Eq. (3) as a set of ordinary differential equations in $r$ and $\theta$, respectively:

$$
\begin{aligned}
& \frac{1}{r} \frac{d}{d r}\left(r \frac{d G_{m n}}{d r}\right)+\left(\zeta_{m}^{2}(r)-\frac{\eta_{m n}^{2}}{r^{2}}\right) G_{m n}=0, \\
& \frac{d^{2} F_{m n}}{d \theta^{2}}+\eta_{m n}^{2} F_{m n}=0,
\end{aligned}
$$

where the perfect separation is due to the fact that $\zeta_{m}$ is only a function of $r$. Recall that $\zeta_{m}$ is the horizontal wavenumber of vertical mode $m$ determined from the local sound speed profile according to Eq. (2). Also, the water column is perturbed by the internal waves, so the wavenumber $\zeta_{m}$ inside and outside the internal waves will be different.

Because the internal waves in the model are open (not a ring), and the reflection of sound from the terminations of internal wave ducts is insignificant (Lin et al., 2009), there are no modes in the angular direction. However, due to the presence of the internal wavefronts separating areas of different $\zeta_{m}$, there are horizontal modes in the radial direction. Equation (4a) is the Bessel equation and its general solutions are Hankel functions of the first and second kinds, $H_{\eta_{m n}}^{(1,2)}\left(\zeta_{m} r\right)$, representing the outgoing and incoming waves, respectively. Note that $\eta_{m n}$ is the angular wavenumber determining the order of the Hankel function, and the subscript $n$ indicates the horizontal mode number. The angular wavenumber $\eta_{m n}$ and the coefficients of the outgoing and incoming wave components are determined from the interface conditions at the internal wavefronts, and the horizontal mode function $G_{m n}(r)$ is a linear combination of $H_{\eta_{m n}}^{(1,2)}\left(\zeta_{m} r\right)$ with the determined coefficients. The interface conditions will be introduced later in the paper.

Since the final solution of Eq. (4b) is proportional to $\exp \left( \pm i \eta_{m n} \theta\right)$, the amplitude $A_{m}$ of the vertical mode is proportional to $G_{m n}(r) \times \exp \left( \pm i \eta_{m n} \theta\right)$. Thus, the horizontal mode $G_{m n}(r)$ will propagate along the internal waves in the $\theta$ direction with the angular wavenumber, $\eta_{m n}$, and its angular cycle distance, $\Theta_{m n}$, along the wavefront can be determined from the following equation:

$$
\Theta_{m n}=\frac{2 \pi}{\eta_{m n}-\eta_{m, n+1}} .
$$

\section{3-D PE approximation method}

The PE approximation method, first introduced by Tappert (1974) to underwater acoustics, is appropriate for analyzing a sound field where forward propagation dominates backward scattering. A Cartesian 3-D PE numerical program (Duda, 2006) is used in this paper to calculate the full acoustic field in the examples. Because it is a full field calculation, the adiabatic mode assumption is not applied. Brief discussion of this numerical method is provided in this section.

Computation of this Cartesian 3-D PE program starts from the source location and marches outward in one direction. At each marching step, the split-step Fourier technique (Hardin and Tappert, 1973) is used to solve the one-way wave equation of parabolic type with a wide-angle approximation to the square-root Helmholtz operator (Feit and Fleck, 1978). The split-step Fourier technique divides sound propagation over a heterogeneous sound speed field into step-by-step free space propagation with a fixed reference wavenumber, and periodically introduces (at each step) phase fluctuations consistent with departures from that fixed reference wavenumber. The free space propagation is handled in the wavenumber domain, and phase anomalies are introduced in the spatial domain. Thus, the computation requires two dimensional (2-D) Fourier transforms. Readers are referred to Jensen et al. (1994) and Duda (2006) for further discussion.

\section{ANALYSIS OF HORIZONTAL MODES}

The interface conditions on curved internal wavefronts will be introduced first, followed by an example of reflection and transmission of Hankel function waves. Then, the characteristic equation of the horizontal modes in curved internal wave acoustic ducts is derived. Two types of horizontal ducting can be found, and the general patterns of the horizontal modes are discussed.

\section{A. Interface conditions}

Figure 2 illustrates the reflection and transmission of Hankel function waves of order $\eta$ across a circular interface located at $r=r_{i}$. The mode-number subscripts $m$ and $n$ are dropped in the following discussion for convenience. In Area I where $r<r_{i}$, the horizontal wavenumber is $\zeta_{\mathrm{I}}$, and in

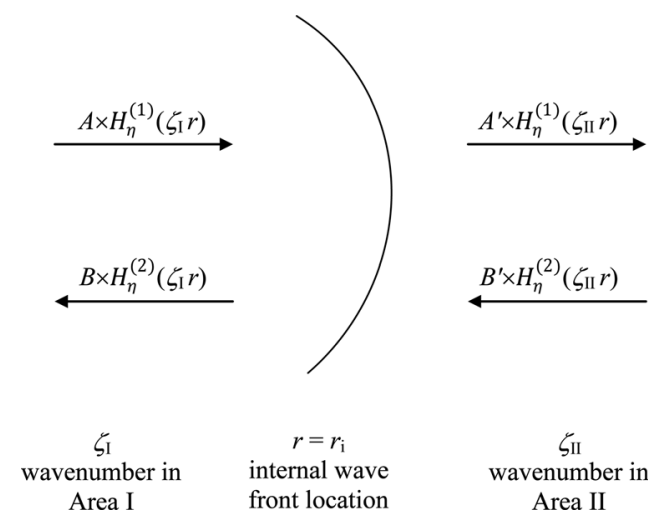

FIG. 2. Reflection and transmission of Hankel function waves of higher order across a circular interface at $r=r_{\mathrm{i}}$. 
Area II where $r>r_{i}$, it is $\zeta_{\mathrm{II}}$. Also, the total wave field is $G_{\mathrm{I}}(r)=A H_{\eta}^{(1)}\left(\zeta_{\mathrm{I}} r\right)+B H_{\eta}^{(2)}\left(\zeta_{\mathrm{I}} r\right)$ in Area I, and is $G_{\mathrm{II}}(r)$ $=A^{\prime} H_{\eta}^{(1)}\left(\zeta_{\mathrm{II}} r\right)+B^{\prime} H_{\eta}^{(2)}\left(\zeta_{\mathrm{II}} r\right)$ in Area II. One way to consider the relationship among these wave components is that $B H_{\eta}^{(2)}\left(\zeta_{\mathrm{I}} r\right)$ is the reflection of an incident wave $A H_{\eta}^{(1)}\left(\zeta_{\mathrm{I}} r\right)$ onto the interface, with an incoming wave $B^{\prime} H_{\eta}^{(2)}\left(\zeta_{\mathrm{II}} r\right)$ from the outer area and a transmitting wave $A^{\prime} H_{\eta}^{(1)}\left(\zeta_{\mathrm{II}} r\right)$ outward.

The interface conditions are derived by requiring continuity and smoothness for the solution across the interface, i.e., $G_{\mathrm{I}}\left(r_{\mathrm{i}}\right)=G_{\mathrm{II}}\left(r_{\mathrm{i}}\right)$ and $d G_{\mathrm{I}} /\left.d r\right|_{r=r_{i}}=d G_{\mathrm{II}} /\left.d r\right|_{r=r_{i}}$. After straightforward but exhaustive algebraic manipulation using the recurrence relation of Hankel functions (Abramowitz and Stegun, 1964), i.e.,

$$
\frac{d}{d r} H_{\eta}^{(1,2)}(\zeta r)=-\zeta H_{\eta+1}^{(1,2)}(\zeta r)+\frac{\eta}{r} H_{\eta}^{(1,2)}(\zeta r)
$$

one can find a linear relation for the coefficients in $G_{I}$ and $G_{\mathrm{II}}$ as shown in the next equation,

$$
\left[\begin{array}{l}
A^{\prime} \\
B^{\prime}
\end{array}\right]=Q_{2 \times 2}\left(\eta, \zeta_{\mathrm{I}}, \zeta_{\mathrm{II}}, r_{i}\right)\left[\begin{array}{l}
A \\
B
\end{array}\right]
$$

where $Q$ is a $2 \times 2$ matrix depending on $\eta, \zeta_{\mathrm{I}}, \zeta_{\mathrm{II}}$, and $r_{i}$, and its detailed formula is

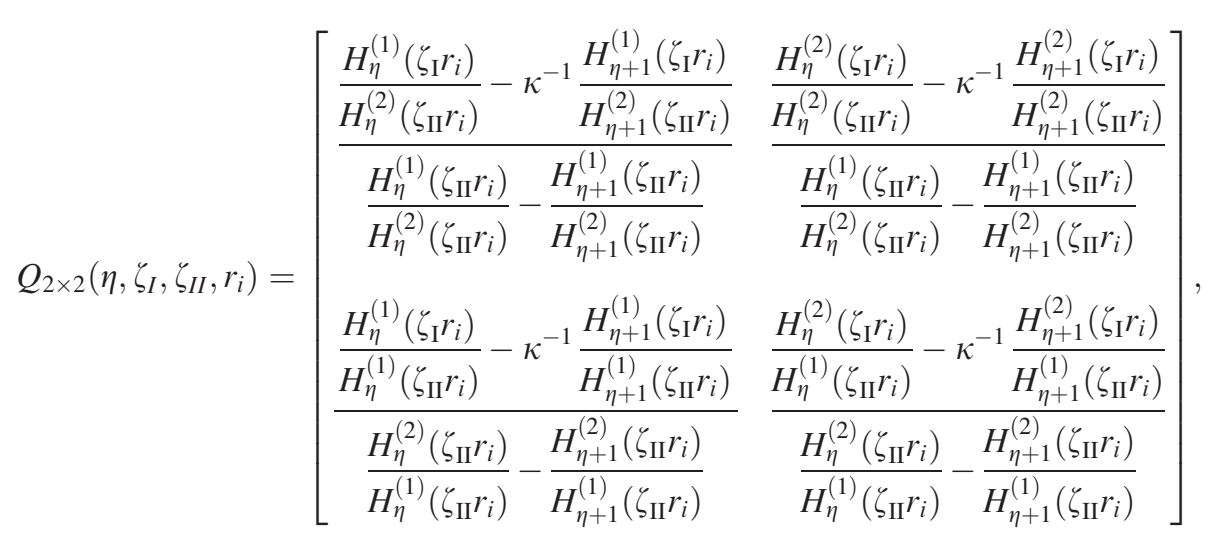

where $\kappa$ is the index of refraction defined as $\kappa=\zeta_{\mathrm{II}} / \zeta_{\mathrm{I}}$. One can also show that when another pair of independent Bessel functions, i.e., the Bessel functions of the first and second kinds $J_{\eta}(\zeta r)$ and $Y_{\eta}(\zeta r)$ is used to construct the general solution of Eq. (4a), the relation between wave components will have the same form as Eqs. (7), with only the two Hankel functions replaced by the two Bessel functions. This formulation of the reflection and transmission of Hankel function waves across a single interface will be used later to construct the characteristic equation for the horizontal modes in the radial direction, which are called radial modes hereafter.

Next we use Eqs. (7) to examine the reflection coefficient of a Hankel function wave incident on a circular interface. Let $A$ equal one and $B^{\prime}$ equal zero, and we can solve the equation for $B$, which is the reflection coefficient $R$ :

$$
R=-\frac{\frac{H_{\eta}^{(1)}\left(\zeta_{\mathrm{II}} r_{i}\right)}{H_{\eta+1}^{(1)}\left(\zeta_{\mathrm{II}} r_{i}\right)}-\kappa \frac{H_{\eta}^{(1)}\left(\zeta_{\mathrm{I}} r_{i}\right)}{H_{\eta+1}^{(1)}\left(\zeta_{\mathrm{I}} r_{i}\right)}}{\frac{H_{\eta}^{(1)}\left(\zeta_{\mathrm{II}} r_{i}\right)}{H_{\eta+1}^{(1)}\left(\zeta_{\mathrm{II}} r_{i}\right)}-\kappa \frac{H_{\eta}^{(2)}\left(\zeta_{\mathrm{I}} r_{i}\right)}{H_{\eta+1}^{(2)}\left(\zeta_{\mathrm{I}} r_{i}\right)}} \times \frac{H_{\eta+1}^{(1)}\left(\zeta_{\mathrm{I}} r_{i}\right)}{H_{\eta+1}^{(2)}\left(\zeta_{\mathrm{I}} r_{i}\right)} .
$$

Using the asymptotic formulas of Bessel functions for large real order, i.e., $J_{\eta}(x) \sim(1 / \sqrt{2 \pi \eta})(e x / 2 \eta)^{\eta}$ and $Y_{\eta}(x) \sim-\sqrt{(2 / \pi \eta)}(e x / 2 \eta)^{-\eta}$, where $e$ is the base of the natural logarithm (Abramowitz and Stegun, 1964), we can obtain the asymptotic behavior of the reflection coefficient for large real $\eta$. Note that $H_{\eta}^{(1)}=J_{\eta}(x)+i Y_{\eta}(x)$ and $H_{\eta}^{(2)}=J_{\eta}(x)$ - $i Y_{\eta}(x)$. Thus, $H_{\eta+1}^{(1)}\left(\zeta_{\mathrm{I}} r_{i}\right) / H_{\eta+1}^{(2)}\left(\zeta_{\mathrm{I}} r_{i}\right)$ is asymptotic to -1 , and both $H_{\eta}^{(1)}\left(\zeta_{\mathrm{I}} r_{i}\right) / H_{\eta+1}^{(1)}\left(\zeta_{\mathrm{I}} r_{i}\right)$ and $H_{\eta}^{(2)}\left(\zeta_{\mathrm{I}} r_{i}\right) / H_{\eta+1}^{(2)}\left(\zeta_{\mathrm{I}} r_{i}\right)$ are asymptotic to $\zeta_{\mathrm{I}} r_{i} / 2 \eta$. The reflection coefficient $R$ is asymptotic to but never equal to one. On the other hand, the asymptotes of Hankel functions for small real order are $H_{\eta}^{(1)}(x) \sim \sqrt{2 /(\pi x)}$ $\times \exp (i(x-\eta \pi / 2-\pi / 4))$ and $H_{\eta}^{(2)}(x) \sim \sqrt{2 /(\pi x)} \exp (-i(x$ $-\eta \pi / 2-\pi / 4)$ ), so the magnitude of the reflection coefficient

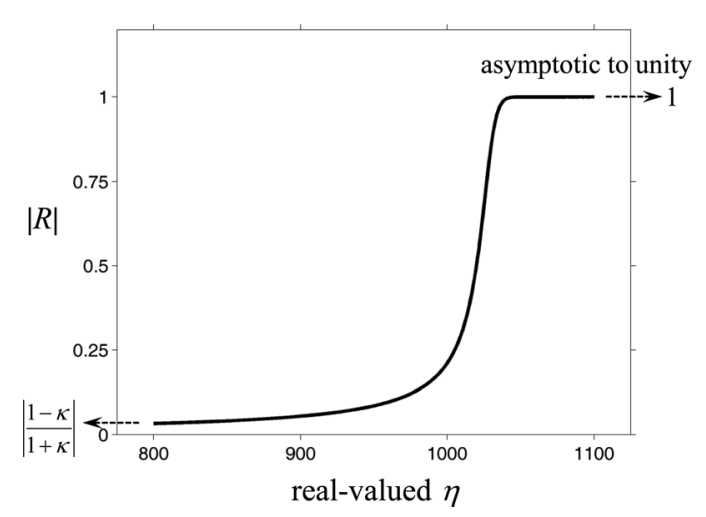

FIG. 3. Reflection coefficient of a Hankel function wave as a function of real $\eta$. Calculation uses Eq. (8) with $\zeta_{\mathrm{I}}=0.2094 \mathrm{~m}^{-1}$ and $\zeta_{\mathrm{II}}=0.2040 \mathrm{~m}^{-1}$. The interface is at $r_{\mathrm{i}}=5 \mathrm{~km}$. 
for a small and real $\eta$ is $|R| \sim|(1-\kappa) /(1+\kappa)|$, which is also the limit of the plane wave reflection (Frisk, 1994).

An example calculation of the reflection coefficient magnitude $|R|$ is shown in Fig. 3 for $\kappa<1$, and confirms the asymptotic behavior discussed above. More importantly, the calculation shows that the reflection coefficient gradually approaches the asymptote with increasing real $\eta$, so total reflection $(R=1)$ will never occur for real $\eta$. This observation is important because it will lead to complex eigenvalues for the radial modes.

\section{B. Characteristic equation of radial modes}

Solving Eq. (4a) for the radial modes requires the horizontal wavenumber of vertical modes, $\zeta_{m}$, determined from Eq. (2) along the radial. Because the water column is perturbed by the internal waves, the horizontal wavenumber $\zeta_{m \mathrm{I}}$ in the area outside the waves (Area I) will be different from the horizontal wavenumber $\zeta_{m I I}$ inside the waves (Area II). The left panel of Fig. 4 is a horizontal plane showing these two different areas, and since internal waves of depression are considered, $\zeta_{m I I}$ is smaller than $\zeta_{m I}$. There are in fact four wavefronts in this idealized square wave model (two for each internal wave). To solve for the radial modes, interface conditions at all these fronts need to be satisfied. For convenience, an additional model simplification is made for the internal wave width to be effectively infinite so that there will only be two remaining interfaces, as shown in the right panel of Fig. 4. This is called the well approximation in literature (Oba and Finette, 2002; Finette and Oba, 2003; McMahon et al., 2012) without considering the horizontal tunneling of sound through internal waves (Lin et al., 2009). Some discussion will be provided on the tunneling effect in Sec. IV B.

One can use the reflection and transmission of Hankel function waves shown in Eqs. (7) to construct a characteristic equation for determining the eigenvalues $\eta_{m n}$ of the radial modes. To simplify the notation, the subscripts $m$ and $n$ of mode numbers are dropped in the following discussion. As shown in Fig. 5, with the well approximation there are six components of Hankel-function waves constituting the radial modes in the simplified model. The inner front is at $r=D$, and the width of the duct is $W$. So the outer front is at $r=D$ $+W$. The wave components in each area are shown, and their coefficients and the eigenvalue $\eta$ need to be determined from the boundary and interface conditions.

Equations (7) are derived from conditions at a single interface, and for the case shown here relationships between wave components across the duct can be determined by cascading the interface conditions, i.e.,

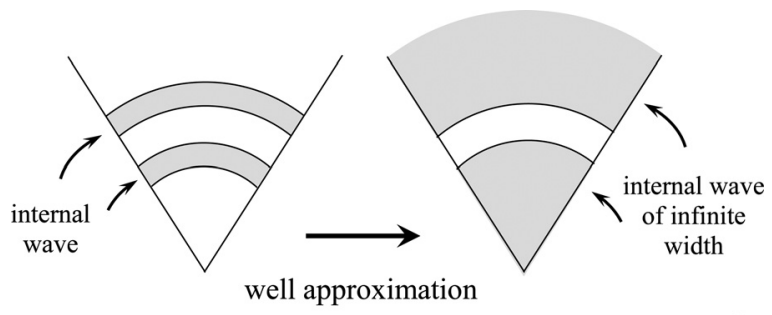

FIG. 4. An internal wave duct and the well approximation.

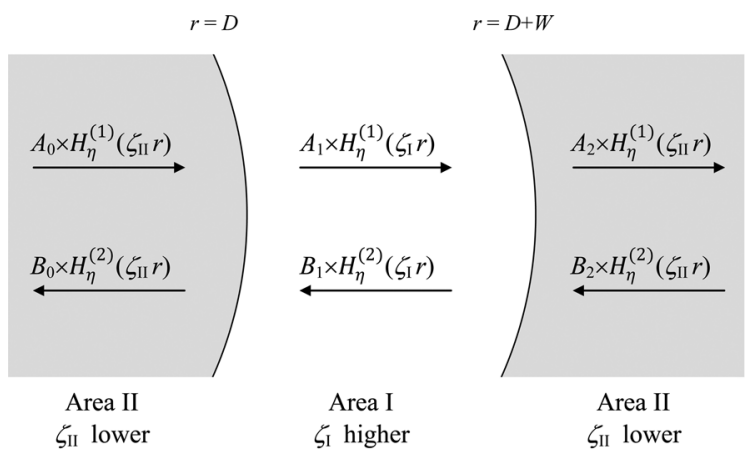

FIG. 5. Wave components along the radial axis in a curved duct.

$$
\begin{aligned}
{\left[\begin{array}{l}
A_{2} \\
B_{2}
\end{array}\right] } & =Q_{2 \times 2}\left(\eta, \zeta_{\mathrm{I}}, \zeta_{\mathrm{II}}, D+W\right)\left[\begin{array}{l}
A_{1} \\
B_{1}
\end{array}\right] \\
& =Q_{2 \times 2}\left(\eta, \zeta_{\mathrm{I}}, \zeta_{\mathrm{II}}, D+W\right) Q_{2 \times 2}\left(\eta, \zeta_{\mathrm{II}}, \zeta_{\mathrm{I}}, D\right)\left[\begin{array}{l}
A_{0} \\
B_{0}
\end{array}\right],
\end{aligned}
$$

where the definition of the $2 \times 2$ matrix $Q$ is given in Eq. (7b). In addition to the interface conditions, we also need to employ the boundary conditions at $r=0$ and $\infty$. Since the radial modes need to be finite at $r=0, B_{0}$ must be equal to $A_{0}$ so that the solution in $r<D$ can be the Bessel function of the first kind, $J_{\eta}\left(\zeta_{\text {II }} r\right)$, which is finite at $r=0$. Also, in order to satisfy the Sommerfeld radiation boundary condition at infinite $r, B_{2}$ must be zero [no incoming wave $H_{\eta}^{(2)}\left(\zeta_{\mathrm{II}} r\right)$ from $r>D+W]$. Substituting these two requirements into Eq. (9) gives

$$
\begin{aligned}
& {\left[\begin{array}{l}
A_{1} \\
B_{1}
\end{array}\right]=Q_{2 \times 2}\left(\eta, \zeta_{\mathrm{II}}, \zeta_{\mathrm{I}}, D\right)\left[\begin{array}{l}
A_{0} \\
A_{0}
\end{array}\right],} \\
& {\left[\begin{array}{c}
A_{2} \\
0
\end{array}\right]=Q_{2 \times 2}^{s q}\left(\eta, \zeta_{\mathrm{I}}, \zeta_{\mathrm{II}}, D, W\right)\left[\begin{array}{l}
A_{0} \\
A_{0}
\end{array}\right],}
\end{aligned}
$$

where $\zeta_{\mathrm{I}}, \zeta_{\mathrm{II}}, D$, and $W$ are known quantities, and a new variable $Q^{s q}\left(\eta, \zeta_{\mathrm{I}}, \zeta_{\mathrm{II}}, D, W\right)$ is defined to represent $Q\left(\eta, \zeta_{\mathrm{I}}\right.$, $\left.\zeta_{\mathrm{II}}, D+W\right) \times Q\left(\eta, \zeta_{\mathrm{II}}, \zeta_{\mathrm{I}}, D\right)$. Equations (10) provide how $A_{1}, B_{1}$, and $A_{2}$ relate to $A_{0}$. Note that $A_{0}$ will be determined ultimately through normalization of the mode functions, which is presented in Appendix A. The last equality of Eq. (10b) can yield the characteristic equation for the eigenvalue $\eta$

$$
q_{21}^{s q}\left(\eta, \zeta_{\mathrm{I}}, \zeta_{\mathrm{II}}, D, W\right)+q_{22}^{s q}\left(\eta, \zeta_{\mathrm{I}}, \zeta_{\mathrm{II}}, D, W\right)=0,
$$

where $q_{j k}^{s q}$ is the element of the matrix $Q^{s q}$ at the $j$ th row and the $k$ th column. It is probably impossible to derive a closedform solution for the eigenvalue $\eta$ with given $\zeta_{\mathrm{I}}, \zeta_{\mathrm{II}}, D$, and $W$, but Eq. (11) can still be solved by numerical root finders. It is because Hankel functions are analytic with respect to their order and so are differentiable with respect to $\eta$ (Abramowitz and Stegun, 1964).

The discussion on the reflection coefficient from a single interface in Sec. III A leads to an important property for the characteristic equation: its solution for the radial mode 
eigenvalue $\eta$ is complex-valued even when the vertical mode eigenvalue $\zeta$ is real-valued. This comes from the fact that when $\eta$ is a real number, no total reflection will occur, see Sec. III A, and hence no mode can form. In addition, because the curved internal wavefronts are soft interfaces (sound can penetrate them), the guided sound propagating along the curved internal wave duct in the $\theta$ direction will encounter radiation damping/losses (Carlstone, 1992). Thus, the angular wavenumber $\eta$ of the radial mode must be complex to describe the decay of sound due to that effect.

The effects of internal wave parameters, such as the index of reflection $\kappa=\zeta_{\mathrm{II}} / \zeta_{\mathrm{I}}$, the radius of curvature $D$ and the gap between internal waves $W$, on the horizontal ducting condition will be studied numerically in Sec. V. Also, it is a future research direction to perform an asymptotic analysis to the characteristic equation.

\section{Types of radial modes}

Once the eigenvalue of the radial mode is determined, we can obtain the radial mode in the duct where $D \leq r \leq D+W$

$$
G_{m n \mathrm{I}}(\mathrm{r})=A_{1} H_{\eta_{m n}}^{(1)}\left(\zeta_{m \mathrm{I}} r\right)+B_{1} H_{\eta_{m n}}^{(2)}\left(\zeta_{m \mathrm{I}} r\right)
$$

where the subscripts $m$ and $n$ are restored to distinguish the vertical and radial mode numbers. The coefficients of the incoming and outgoing components, $A_{1}$ and $B_{1}$, also depend on the internal wave curvature and the duct width, as shown in Eqs. (10), in addition to the wavenumber contrast between $\zeta_{m \mathrm{I}}$ and $\zeta_{m \mathrm{II}}$.

Equation (12) is a general solution of the radial mode within the curved duct, and there are two types of radial trapped modes. The first is the whispering gallery mode formed by the sound continually reflecting off the outer boundary, for which the mode function has peaks close to the outer boundary (Rayleigh, 1910). The second type of radial trapped mode is the fully bouncing mode formed by the sound reflecting between the wavefronts. These two types of modes are depicted in Fig. 6.

Although it is not shown here rigorously, the radial mode shape within the duct can be approximated by the Bes-

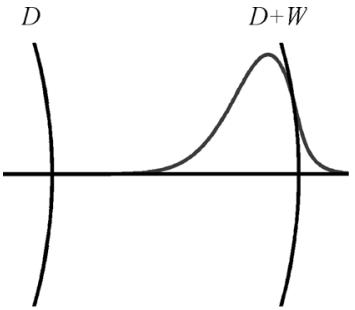

(a) whispering gallery mode

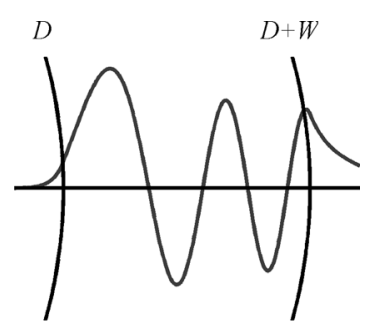

(b) fully bouncing mode
FIG. 6. Two types of radial modes in a curved internal wave duct.

sel function of the first kind, $J_{\eta_{m n}}\left(\zeta_{m \mathrm{I}} r\right)$, which means the coefficients $A_{1}$ and $B_{1}$ in Eq. (12) have very close values. Because a Bessel function descends rapidly to zero when its argument drops below its order, we can compare $\eta_{m n}$ and $\zeta_{m \mathrm{I}} D$ to characterize the mode type, which, in other words, is closely related to the location (at $r=\eta_{m n} / \zeta_{m \mathrm{I}}$ ) of the inflection point of the mode function. When $\eta_{m n}$ is significantly greater than $\zeta_{m \mathrm{I}} D$ (the inflection point is located at $r>D$ ), the corresponding mode is considered a whispering gallery mode; otherwise, it is a fully bouncing mode. This criterion was in fact used by Buckingham (1987) on determining the envelope of refracted sound in a slope environment. In the current application, it essentially characterizes the case of a radial mode in the inner domain where $r<D$. A whispering gallery mode has its tail vanishingly small because the guided sound bounces along the outer boundary only. In contrast, a fully bouncing mode has a noticeable tail indicating that the trapped sound also interacts with the inner boundary.

\section{NUMERICAL ILLUSTRATIONS}

The water depth in the numerical example is $80 \mathrm{~m}$, and the acoustic frequency is $75 \mathrm{~Hz}$. The sound speed profile and the bottom geoacoustic parameters are shown in Fig. 7(a). The thickness of upper higher-speed layer is $20 \mathrm{~m}$ and the amplitude of the internal wave of depression is $20 \mathrm{~m}$. Environmental parameters of the internal wave field are: radius of curvature $D=50 \mathrm{~km}$, gap between the waves (the duct width) $W=500 \mathrm{~m}$, and wave width $w=300 \mathrm{~m}$. Note that the well approximation is employed in calculating the radial modes, but not in the 3-D PE calculation.

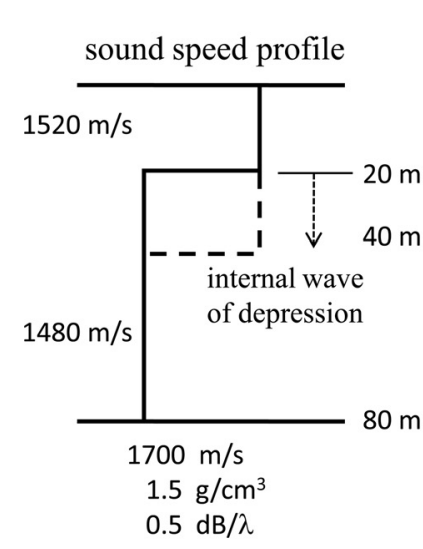

(a)

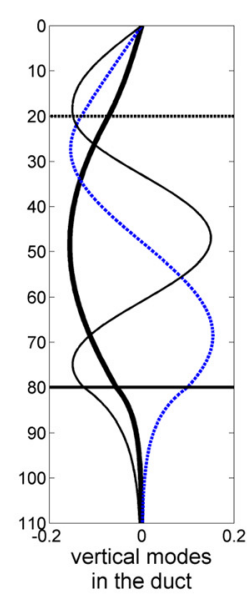

(b)

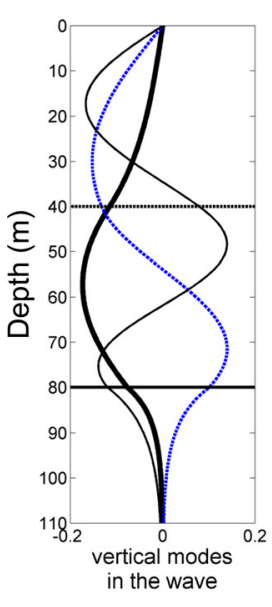

(c)

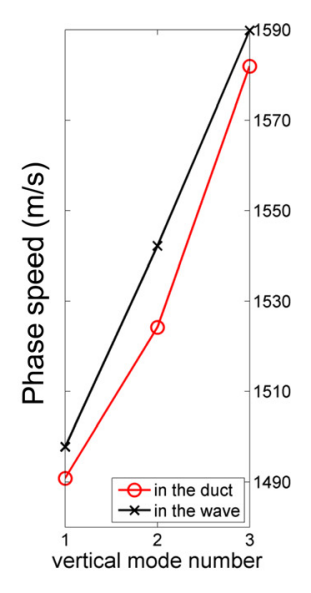

(d)
FIG. 7. (Color online) Vertical modes and their phase speeds in the water column model. (a) Sound speed profiles with and without the internal wave perturbation. The first three vertical modes of $75 \mathrm{~Hz}$ sound (b) inside the duct and (c) inside the wave are shown. (d) Phase speeds of the vertical modes in the duct (circles) and the wave (crosses). 


\section{A. Radial mode calculations}

Calculating radial modes requires information about the vertical modes, which are shown in Figs. 7(b) and 7(c) along with their phase speeds in Fig. 7(d) (the modal attenuations are included but not shown). From the modal phase speeds, one can see that the strongest modal contrast across the wave occurs on the second vertical mode $(m=2)$, which encounters an abrupt change on the sound speed at its upper turning point at depth $\sim 30 \mathrm{~m}$.

A numerical root finder using the bisection method is implemented to solve the characteristic equation (11) for the eigenvalues/angular wavenumbers of the radial modes. Three different horizontal ducting situations are identified: no ducting, whispering gallery type ducting, and full bouncing between the two wavefronts. Accordingly, three corresponding types of radial modes are found: leaky modes, whispering gallery modes, and fully bouncing modes. Discussion on the radial mode solutions is provided below.

Figure 8(a) shows the angular wavenumbers of first eight radial modes for vertical mode 1 in terms of their ratios to the radius of internal wave curvature, i.e., $\eta_{1 n} / D$ with $n=1-8$. The imaginary part of this ratio can be considered as the reciprocal of a modal decay distance over which the amplitude of a radial mode decays by a factor of $e$ along the inner wavefront. The radial mode of the lowest order $(n=1)$ has the slowest decay rate, and the rate increases as the mode number $n$ increases. The modal decay distance is a good indicator of the effectiveness of a radial mode propagating in the curved duct. Considering $10 \mathrm{~km}$ as a cutoff, the first two radial modes shown here are propagating modes, and the others are leaky modes. By the characterization method for radial mode functions based on the locations of their inflection points (see Sec. III C), the two propagating trapped modes are of whispering gallery type. This is because the ratios of their eigenvalues to the radius of internal wave curvature $\eta_{1 n} / D$, where $n=1-2$, are significantly higher than the real part of the vertical mode eigenvalue $\zeta_{1 \mathrm{I}}$, which is about $0.3161 \mathrm{~m}^{-1}$. The mode functions are calculated and plotted in Fig. 8(b), and the first two radial modes are indeed whispering gallery modes. Their angular cycle distances $\Theta_{m n}$, where $m=1$ and $n=1-2$, can be calculated using Eq. (5), and the values are 0.1901 and $0.2142 \mathrm{rad}$, respectively. The cycle distances of these two whispering gallery modes along the outer wavefront, converted into length by $(D+W) \times \Theta_{m n}$, are both of order $10 \mathrm{~km}$, which are smaller than their modal decay distances.

This example also shows that the total number of radial trapped modes depends on the vertical mode number $m$, and the discussion is provided here. Because the phase speed contrast of vertical mode 2 across the wavefront is greatest compared to other vertical modes, it should have more radial trapped modes. This is confirmed in the radial mode calculation results shown in Figs. 8(c) and 8(d). All three types of radial modes, in fact, appear in this case $(m=2)$, and they are whispering gallery modes for $n=1-4$, fully bouncing modes for $n=5-6$, and leaky modes for $n \geq 7$. The cycle distances of the whispering gallery modes are again of order $10 \mathrm{~km}$. Finally, it is worth noting that all of the radial modes seen in this example are complex, while the leaky modes have significantly larger imaginary parts.

\section{B. Full 3-D PE calculation}

A Cartesian 3-D PE numerical program (Duda, 2006) is used to calculate the full acoustic field in the example model without employing the adiabatic mode assumption. Vertical mode filtering is employed to extract the amplitude of modes
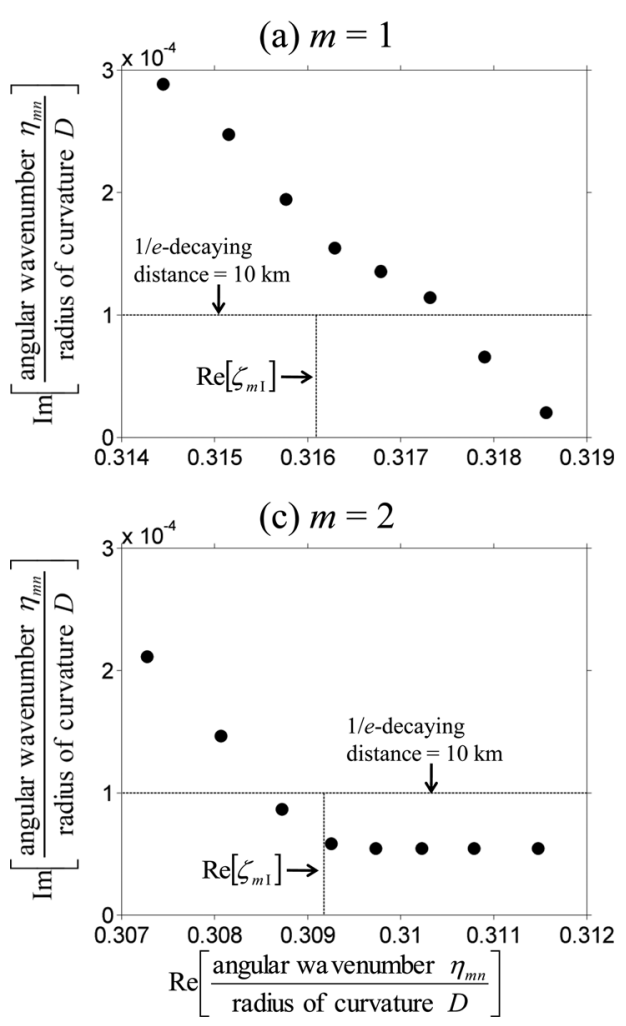

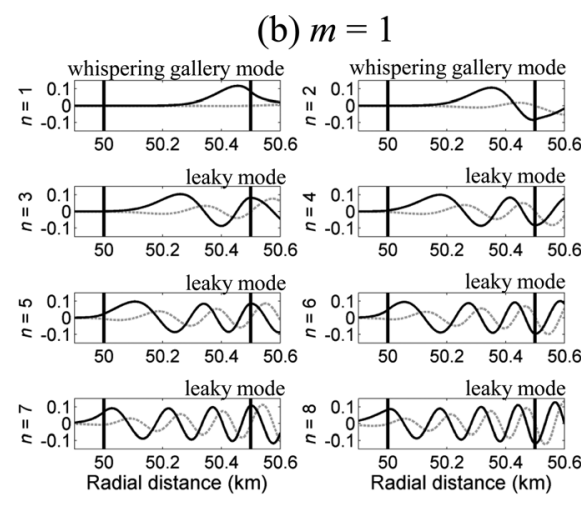

(d) $m=2$
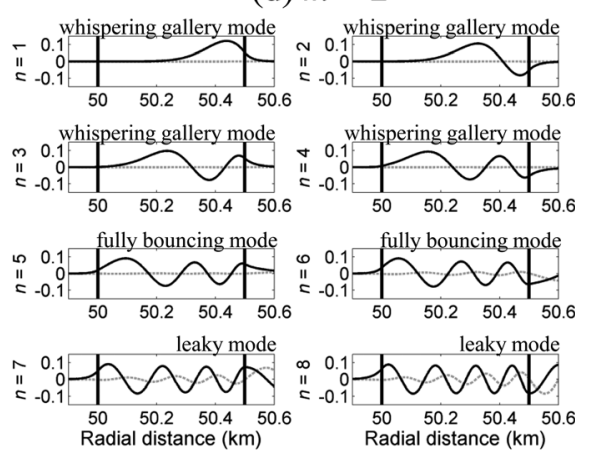

FIG. 8. Radial modes of $75 \mathrm{~Hz}$ sound and the ratios of their angular wavenumbers $\eta_{m n}$ to the radius $D$ of internal wave curvature $(D=50 \mathrm{~km})$. (a) and (b) correspond to vertical mode 1 , and (c) and (d) correspond to vertical mode 2. The solid curves in (b) and (d) are real parts of the radial modes, and dashed curves are imaginary parts. 
(a) vertical mode 1 , source at $y=-175 \mathrm{~m}$

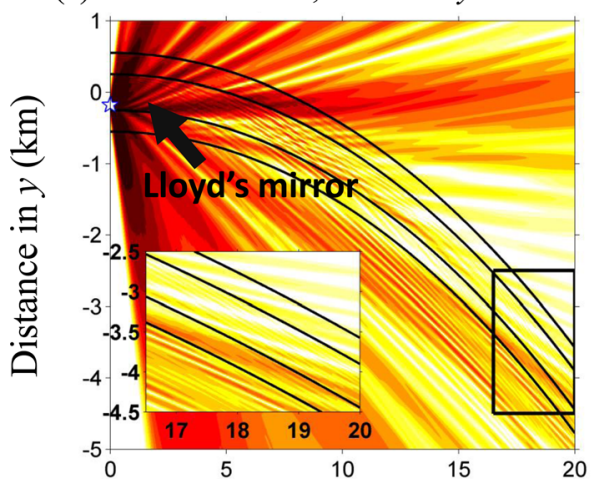

(c) vertical mode 2 , source at $y=-175 \mathrm{~m}$

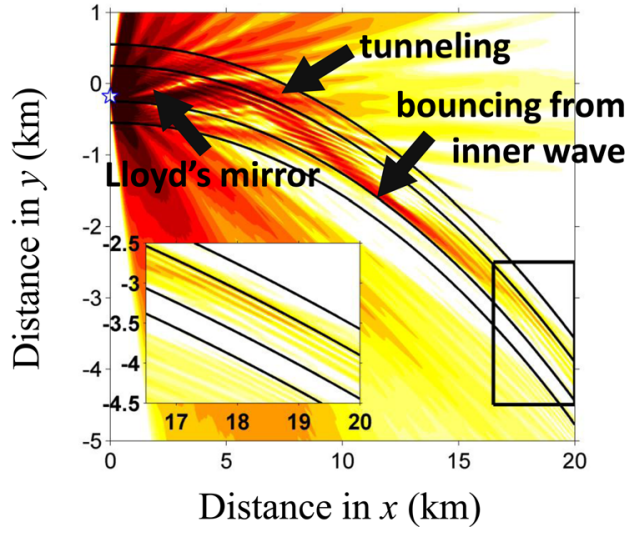

(b) vertical mode 1 , source at $y=175 \mathrm{~m}$

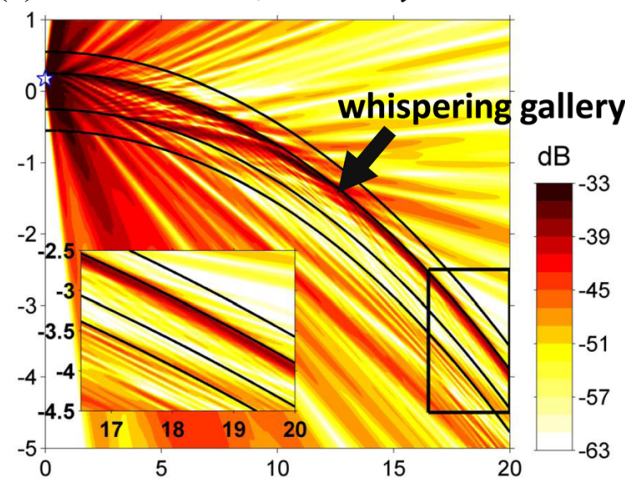

(d) vertical mode 2 , source at $y=175 \mathrm{~m}$

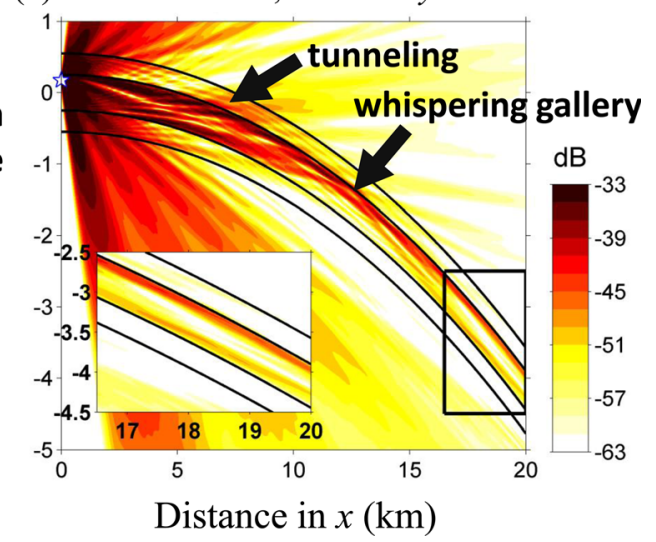

FIG. 9. (Color online) Horizontal ducting of vertical modes 1 and 2 in a curved internal wave duct. The frequency is $75 \mathrm{~Hz}$, and the sound source is placed at depth $65 \mathrm{~m}$. Full 3-D PE solutions with vertical mode filtering are presented in terms of modal intensity. The edges of internal waves are indicated by the solid curves, and the inset panel is a closer view of the area marked by the box.
1 and 2 to examine their horizontal ducting conditions. A point source with $0 \mathrm{~dB}$ intensity measured at $1 \mathrm{~m}$ away is placed at the depth $65 \mathrm{~m}$ and transmits $75 \mathrm{~Hz}$ tonal sound. Two source locations are considered. One source location is inside the duct and $75 \mathrm{~m}$ away from the outer wavefront. The other is also inside the duct, but it is $75 \mathrm{~m}$ away from the inner wave. When the origin of the horizontal coordinates $(x, y)$ is placed at the center of the $500 \mathrm{~m}$ wide duct, the two source locations are at $y= \pm 175 \mathrm{~m}$. The excitation of vertical modes 1 and 2 is about $-6 \mathrm{~dB}$ and $-4 \mathrm{~dB}$, respectively, at $1 \mathrm{~m}$ from the source.

The radial modes shown in Fig. 8 indeed capture the horizontal ducting seen in the 3-D PE solution. For example, the modal theory predicts that vertical mode $1(m=1)$ has only corresponding radial trapped modes of the whispering gallery type, and the 3-D PE solution in Figs. 9(a) and 9(b) indeed shows that only when the source is located close to the outer wave, vertical mode 1 is trapped. The modal theory also predicts that vertical mode $2(m=2)$ will encounter strong horizontal ducting with both whispering gallery and fully bouncing types, and the PE solution in Figs. 9(c) and 9(d) shows that no matter where the source is placed inside the duct, vertical mode 2 can be trapped in the duct.

In addition to the horizontal ducting, the 3-D PE solution also shows other interesting propagation effects. First, when the source is located close to the inner wave inside the duct, as shown in Figs. 9(a) and 9(c), the split beam pattern observed near the source on the convex side of the inner wave is caused by the horizontal Lloyd's mirror effect (Badiey et al., 2011; McMahon et al., 2012). Also, vertical mode coupling occurs in the areas near the source where the sound propagates across the internal waves with greater grazing angles, and it causes discontinuity of the acoustic beams across the waves. Last, the 3-D PE solution contains the tunneling effect, which was not seen in the mode calculation due to the well approximation. As shown in Figs. 9(c) and 9(d), when the horizontal ducting starts appearing, some of the trapped sound tunnels through the outer internal wave at locations from $x=6 \mathrm{~km}$ to $12 \mathrm{~km}$. The modal analysis of this tunneling effect has been conducted for straight internal wave ducts (Lin et al., 2009), and the extension to curved ducts is a topic for future research.

\section{DISCUSSION}

Further discussions on the horizontal ducting of sound by curved internal waves are provided in this section. We will first examine the effect of wavefront curvature, and then present a meandering wave case, for which the curvature has a longitudinal variation. A parametric study of ducting conditions will be implemented, and their general pattern will be presented.

A numerical calculation is performed to examine the dependency of the horizontal ducting on internal wave curvature. All the environmental parameters are the same as the example in Sec. IV except for the radius of internal wave curvature, which decreases from $50 \mathrm{~km}$ to $25 \mathrm{~km}$. The radial modes corresponding to vertical mode 2 are shown in Figs. 10(a) and 10(b) for their angular wavenumbers and mode functions. Compared with the case of the $50 \mathrm{~km}$ radius shown in Figs. 8(c) and 8(d), we see that fewer trapped radial 


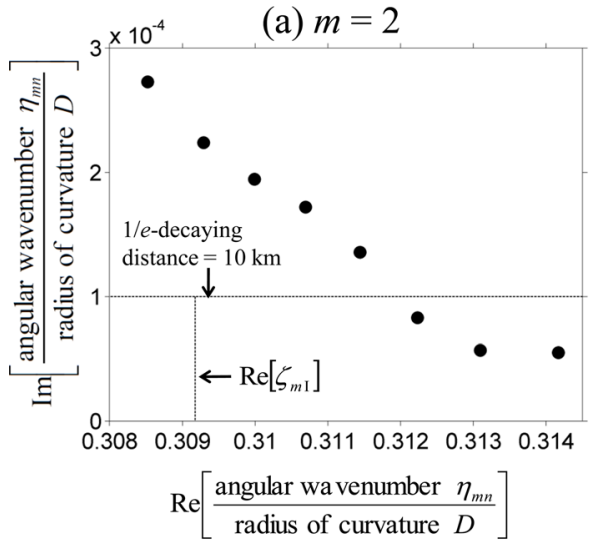

(c) vertical mode 2 , source at $y=-175 \mathrm{~m}$

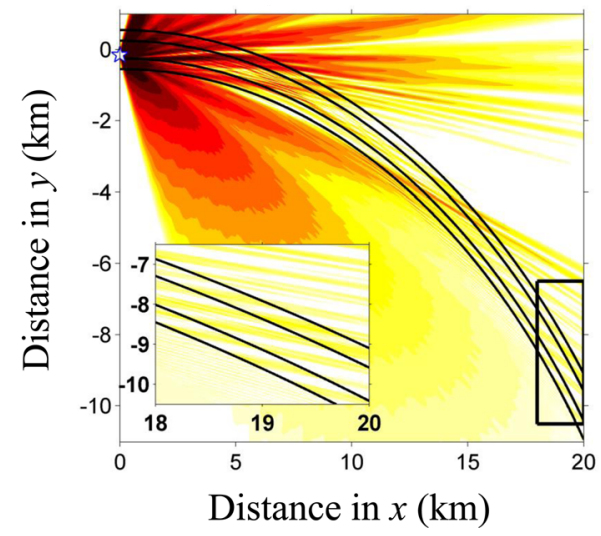

(b) $m=2$
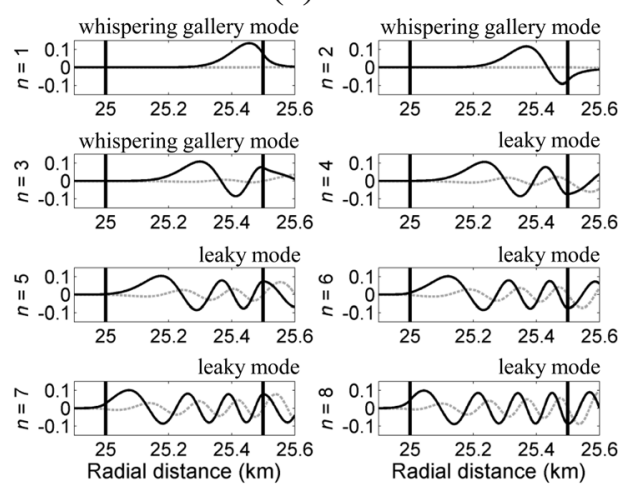

(d) vertical mode 2 , source at $y=200 \mathrm{~m}$

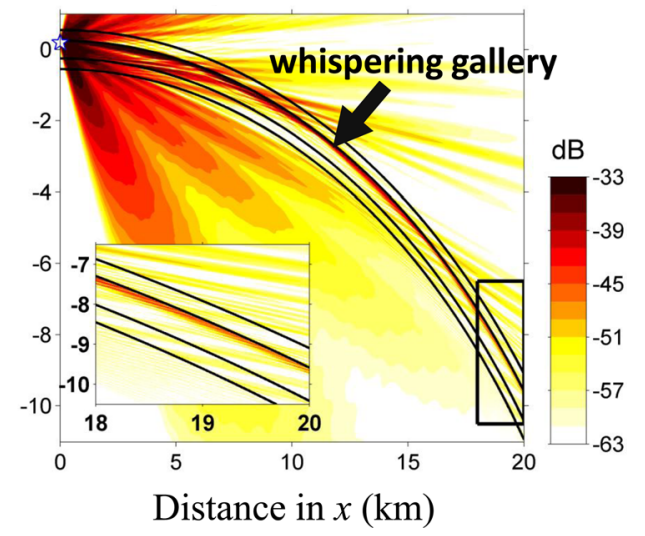

FIG. 10. (Color online) Horizontal ducting of vertical mode 2 at $75 \mathrm{~Hz}$ in a curved internal wave duct. The radius of internal wave curvature is $25 \mathrm{~km}$, which is smaller than the case shown in Figs. 8 and 9. The ratios of the radial mode eigenvalues $\eta_{m n}$ to the radius $D$ of internal wave curvature are shown in (a), and the radial mode functions are shown in (b). The intensity of vertical mode 2 obtained from full 3-D PE calculation with vertical mode filtering are presented. The sound source is placed at the depth $65 \mathrm{~m}$, and two different source locations are considered: (c) near the inner wave and (d) near the outer wave. The edges of internal waves are indicated by the solid curves, and the inset panel is a closer view of the area marked by the box. modes occur in the current case, and they are all whispering gallery modes.

The 3-D PE solutions of vertical mode 2 intensity for two different source locations are shown in Figs. 10(c) and 10(d). In contrast to Figs. 9(c) and 9(d), only when the source is close to the outer wave, vertical mode 2 is trapped in the duct because the horizontal ducting is the whispering gallery type. Also, the cycle distances of these whispering gallery modes are between 6 and $8 \mathrm{~km}$, which are smaller than the values $(10 \mathrm{~km})$ in the preceding case. In summary, this test suggests that the number of radial trapped modes, as well as their cycle distances, gradually decreases when the radius of curvature becomes smaller. On the other hand, as the wavefront curvature decreases and becomes closer to straight, there will be more radial trapped modes. In the limiting case of flat wavefronts, the whispering gallery mode disappears, and there will be only fully bouncing modes.

The second calculation is for horizontal ducting in a meandering wave field, where the wavefronts follow a sinusoidal function with a 200-m amplitude and a 20-km wave length. The internal wave curvature is $50.66 \mathrm{~km}$ at the most curved part, and it changes longitudinally along the duct. As sketched in Fig. 11(a), the radial modes tend to be of whispering gallery type in the most curved part. On the other hand, the radial modes tend to be fully bouncing in the less curved portion because the concavity of the wavefront is not great enough to support whispering gallery ducting. Figures 11(b)-11(e) depict the horizontal ducting of vertical modes 1 and 2 for different source locations obtained from 3-D PE calculations. In this example where the source is placed in the curved part, when the source is close to the outer wave, more sound is trapped in the duct; see Figs. 11(c) and 11(e). This is because the whispering gallery modes in the curved part are excited.

In this meandering wave case, we also notice that the radial modes have a longitudinal variation, because the internal wave curvature changes along the waveguide. In a more realistic situation, where the wave shape varies along the wavefront, the radial mode variation will be even more significant. The consequence is that the ducted sound will encounter radial mode coupling. The 3-D PE modeling technique can be used to study sound propagation in this situation numerically (Duda et al., 2011). For a more analytical approach, the radial mode coupling can also be analyzed with the virtual mode method (Tindle et al., 1976; Williams, 1978). A preliminary research idea is proposed here and the detailed study is suggested for future research. To solve the coupled radial mode equation, we can expand the radial modes at any position along the duct with one set of discrete plus continuum basis modes. Because the tangent of the wavefront changes along the waves, the basis modes are subjected to rotation. The Jacobian caused by the rotation of the basis modes may be needed to determine the transportation of the modal energy. Also, if we think of the continuum as virtual modes (Tindle et al., 1976; Williams, 1978), the coupling to any virtual mode that can retain energy in the duct is apt to be negligible, so that anything past the trapped mode set can be considered to be a complete loss. So we may keep the same set of trapped modes everywhere. Modes near cutoff might be a flaw in this approach, and the study by 
(a)

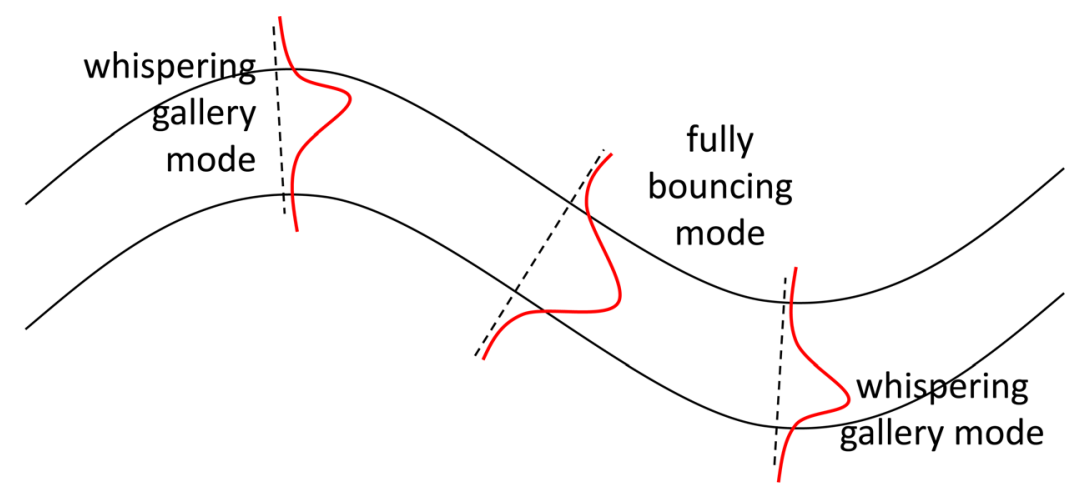

(b) vertical mode 1 , source at $y=-235 \mathrm{~m}$ (c) vertical mode 1 , source at $y=235 \mathrm{~m}$
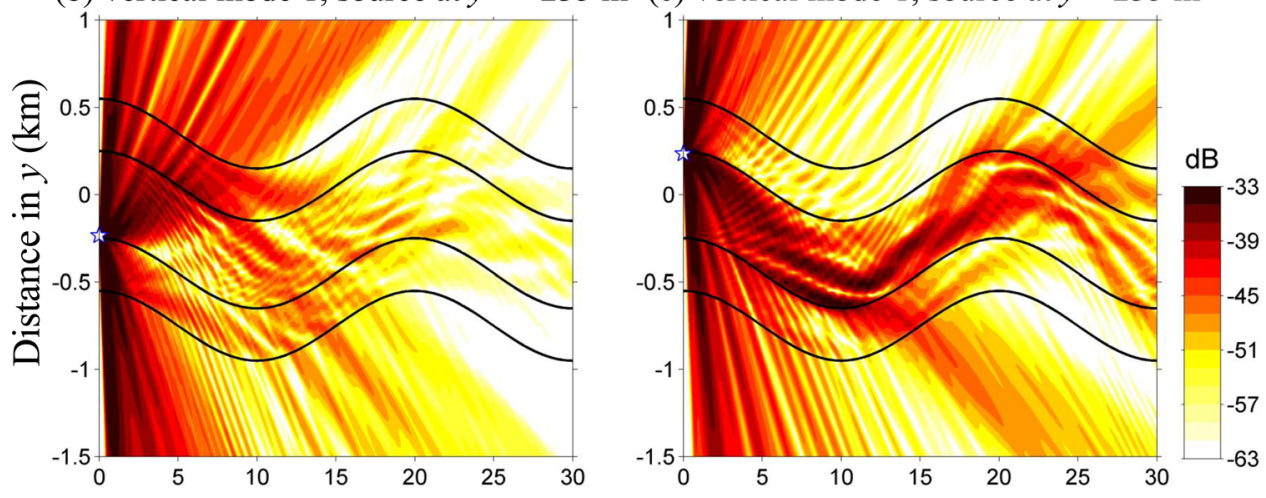

FIG. 11. (Color online) Horizontal ducting of sound in a meandering internal wave duct. (a) A sketch showing different types of ducted modes in the duct. Full 3-D acoustic fields for a source in a curved portion and located (b) near the inner wave, and (c) near the outer wave. (d) Same as (b) except vertical mode 2 is plotted. (e) Same as (c) except vertical mode 2 is plotted.

(d) vertical mode 2 , source at $y=-235 \mathrm{~m}$

(e) vertical mode 2 , source at $y=235 \mathrm{~m}$
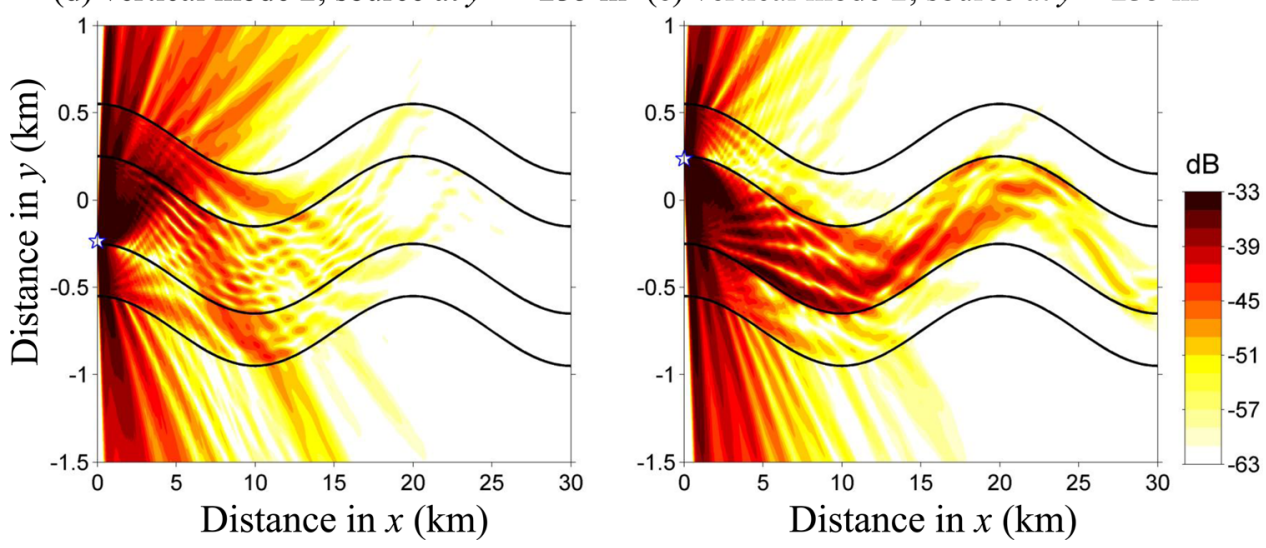

Williams (1978) on the virtual modes in this regime may provide some insight into the problem.

The ducting condition derived from the geometric and ray acoustics approximation by Lynch et al. (2010) has been compared with the normal mode predictions shown above. The ray approach requires the horizontal angle $\theta_{\mathrm{nm}}^{\text {hor }}$ of a radial mode, which can be determined from its eigenvalue $\eta_{m n}$ in the following way:

$$
\theta_{n m}^{\text {hor }}=\cos ^{-1} \frac{\eta_{m n} /\left(D+\frac{W}{2}\right)}{\zeta_{m}},
$$

where the horizontal angle $\theta_{n m}^{\text {hor }}$ is measured at the midpoint of the internal wave duct at $r=D+W / 2$. We also need to use the environmental parameters of internal waves to derive the critical grazing angle for a modal ray reflecting from a wavefront: see Lynch et al. (2010) for details. Then, by comparing $\theta_{n m}^{\text {hor }}$ to the critical angle, we can determine the ducting condition of the mode for three possible outcomes: no ducting,

whispering gallery ducting, and the fully bouncing type of ducting. Ray predictions of the ducting condition mostly agree with results from the normal mode method described earlier in Sec. III C. The exceptions may occur for a small radius of curvature and low vertical mode number $m$, where the ray approximation breaks. For example, with $100 \mathrm{~Hz}$ frequency there may be differences for the radius of curvature $D$ less than $10 \mathrm{~km}$ and for the first vertical mode $(m=1)$.

An analysis using both the ray and normal mode approaches has been conducted to study the dependency of the ducting condition on the radius of internal wave curvature $D$ and the vertical mode number $m$. A sketch of the overall behavior is shown in Fig. 12, and the ducting condition is jointly constrained by $D$ and $m$ such that the boundary of each ducting condition in the dependency diagram has a hyperbolic shape. In general, there is a cutoff vertical mode number for the ducting condition acting like one of the asymptotes of the hyperbolic boundary. Similarly, there is also a maximum curvature (the other asymptote) behind 


\section{Horizontal ducting condition by curved internal waves}

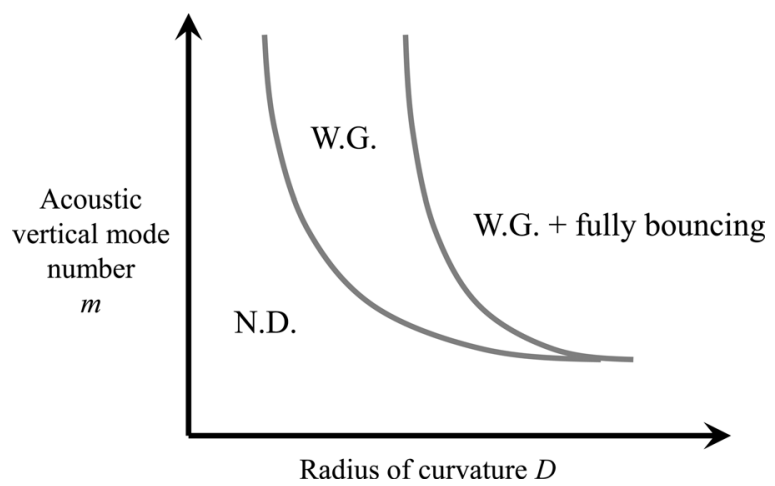

FIG. 12. Dependency of the horizontal ducting condition on the internal wave curvature and the acoustic vertical mode number. N.D. stands for no ducting, and W.G. stands for whispering gallery.

which no sound can be trapped in the duct. In addition, for a given vertical mode, as the radius of curvature varies from small to large, there is generally a sequential change from no ducting (N.D.), to whispering gallery ducting (W.G.), and then to combined ducting by whispering gallery and fully bouncing modes. The same pattern can also be observed for specifying an internal wave curvature and varying the vertical mode number. However, when the curvature is very small, the ducting condition will directly transition to combined ducting. In the limit of straight wavefronts, there will be no whispering gallery modes.

It is also found that as the duct width $W$ increases, the area of the whispering gallery condition in the dependency diagram (Fig. 12) expands with the boundary of the combined ducting moving up and to the right. On the other hand, when the duct width is too small (less than a half of a wavelength), no ducting is permitted. The dependency of the horizontal ducting on acoustic frequency has also been examined. As acoustic frequency increases, the cutoff vertical mode number goes up, and only higher vertical modes can be trapped horizontally in the duct. In fact, higher vertical modes are generally more easily trapped in the curved duct. Finally, although it is not addressed here, from the modal ray analyses and the numerical simulations shown by Lynch et al. (2010) and Duda et al. (2011), the strength of the horizontal ducting also depends on the size of internal waves. Readers are referred to the two papers for detailed discussions.

\section{CONCLUSION}

Horizontal ducting of low-frequency sound by curved internal waves is analyzed with 3 -D normal mode and PE methods in this paper. The results are briefly summarized here.

The horizontal acoustic modes in curved internal wave ducts can be determined from the Bessel equation of complex order. Two types of modes can be found: whispering gallery modes and fully bouncing modes. Horizontal ducting depends on both acoustic and internal-wave parameters. For instance, total number of horizontal trapped modes depends on the vertical mode number, and the number gradually decreases as the internal wave curvature becomes greater. On the other hand, as the wave curvature decreases, more horizontal modes occur. Detailed discussion has been provided with a parametric study.

A 3-D normal mode theory is employed to investigate the ducted sound field with the well approximation, and the effect of sound tunneling through internal waves and radiating outward is neglected in the modal analysis. The 3-D full field calculated from the PE method does show the tunneling effect, as well as other effects caused by internal waves but neglected in the modal analysis. It is suggested for future research to avoid the well approximation in the mode calculation so that the tunneling modes can be resolved and investigated within normal mode framework. The mode coupling and horizontal Lloyd's interference seen in the 3-D PE calculations are as important as the ducting, and they all contribute to the acoustic fluctuations in a curved internal wave field.

\section{ACKNOWLEDGMENTS}

This work was sponsored by the Office of Naval Research under grants N00014-10-1-0040 and N00014-111-0701. The authors also thank Dr. Alexey A. Shmelev for providing his numerical mode filtering program.

\section{APPENDIX: NORMALIZATION OF RADIAL MODES}

This appendix concerns the normalization factor of radial modes, i.e., the value of $A_{0}$ in Eqs. (10). The derivation follows closely the normalization of vertical modes shown by Porter (1991). First, we include a point-source forcing function in Eq. (3), i.e.,

$$
\frac{1}{r} \frac{\partial}{\partial r}\left(r \frac{\partial A}{\partial r}\right)+\frac{1}{r^{2}} \frac{\partial^{2} A}{\partial \theta^{2}}+\zeta^{2}(r) A=-\frac{\delta\left(r-r_{0}\right)}{r} \delta(\theta),
$$

where the horizontal wavenumber $\zeta$ is assumed to be only a function of $r$, and the source is placed between two interfaces located at $r=a$ and $b$. The horizontal wavenumber for $a \leq r \leq b$ is $\zeta_{\mathrm{I}}$, and is $\zeta_{\mathrm{II}}$ elsewhere. With the wavenumber integration technique (Frisk, 1994), one can find the following solution to Eq. (A1):

$$
A(r, \theta)=\frac{1}{2 \pi} \int_{-\infty}^{\infty} G(r, \eta) \exp (i \eta \theta) d \eta
$$

where $\eta$ is the angular wavenumber, and $G$ is governed by the next ordinary differential equation with two mixed boundary conditions imposed at $r=a$ and $b$,

$$
\begin{aligned}
& \frac{1}{r} \frac{d}{d r}\left(r \frac{d G}{d r}\right)+\left(\zeta^{2}-\frac{\eta^{2}}{r^{2}}\right) G=-\frac{\delta\left(r-r_{0}\right)}{r}, \\
& \left\{\begin{array}{l}
\frac{f(a, \eta)}{g(a, \eta)} G(a, \eta)+\frac{d G(a, \eta)}{d r}=0 \\
\frac{f(b, \eta)}{g(b, \eta)} G(b, \eta)+\frac{d G(b, \eta)}{d r}=0 .
\end{array}\right.
\end{aligned}
$$


The functions $f(r, \eta)$ and $g(r, \eta)$ in Eq. (A4) actually determine the impedance conditions at the boundarie, and they are, in general, complex-valued. Therefore, the current boundary value problem is not a proper Sturm-Liouville problem, which requires the coefficients of a mixed boundary condition to be real constants. This prevents us from employing Sturm-Liouville theory to solve Eq. (A3) with eigenfunction expansions. Nevertheless, we can still use the endpoint method (Frisk, 1994) to solve for $G$, and then approximate the wavenumber integral solution of $A(r, \theta)$ shown in Eq. (A2) as a modal solution by applying Cauchy's integral theorem and neglecting the branch line integral. It is the goal of this appendix to normalize these modes so that the modal solution can be written in the following form:

$$
A(r, \theta) \cong i \sum_{n} G_{n}\left(r_{0}, \eta_{n}\right) G_{n}\left(r, \eta_{n}\right) \exp \left(i \eta_{n} \theta\right),
$$

where $G_{n}\left(r, \eta_{n}\right)$ is the $n$th normalized radial mode with the modal eigenvalue $\eta_{n}$.

We now employ the end-point method (Frisk, 1994) to solve Eq. (A3) for the wavenumber spectrum $G(r, \eta)$ into the next formulation

$$
G(r, \eta)= \begin{cases}-\frac{1}{C} u_{a}(r, \eta) u_{b}\left(r_{0}, \eta\right), & a \leq r \leq r_{0}, \\ -\frac{1}{C} u_{a}\left(r_{0}, \eta\right) u_{b}(r, \eta), & r_{0} \leq r \leq b,\end{cases}
$$

where the denominator $C=r_{0} W\left[u_{a}\left(r_{0}, \eta\right), u_{b}\left(r_{0}, \eta\right)\right]$, and the symbolic notation $W$ denotes the Wronskian, i.e.,

$$
\begin{aligned}
W\left[u_{a}\left(r_{0}, \eta\right), u_{b}\left(r_{0}, \eta\right)\right]= & u_{a}\left(r_{0}, \eta\right) u_{b}^{\prime}\left(r_{0}, \eta\right) \\
& -u_{a}^{\prime}\left(r_{0}, \eta\right) u_{b}\left(r_{0}, \eta\right) .
\end{aligned}
$$

In Eq. (A7), the prime symbol denotes the first derivative with respect to $r$, and the $u_{a}$ and $u_{b}$ are the solutions of the following two ordinary different equations, respectively:

$$
\begin{aligned}
\mathcal{L}(\eta) u_{a} & =0 ; \quad \mathcal{B}(a, \eta) u_{a}=0, \\
\mathcal{L}(\eta) u_{b} & =0 ; \quad \mathcal{B}(b, \eta) u_{b}=0
\end{aligned}
$$

where the symbol $\mathcal{L}(\eta)$ indicates the left-hand side of Eq. (A3), and the symbol $\mathcal{B}(r, \eta)$ represents the boundary condition shown in Eq. (A4). Note that the horizontal wavenumber $\zeta$ is known so that $\mathcal{L}$ and $\mathcal{B}$ depend on $\eta$ and $r$ only. Substituting the end-point solution of $G(r, \eta)$ into Eq. (A2) yields

$$
A(r, \theta)=\frac{1}{2 \pi} \int_{-\infty}^{\infty} \frac{u_{a}\left(r^{<}, \eta\right) u_{b}\left(r^{>}, \eta\right)}{-C} \exp (i \eta \theta) d \eta,
$$

where the variable $r^{<}$denotes the smaller value between $r$ and $r_{0}$, and $r^{>}$denotes the larger value between $r$ and $r_{0}$. To determine the integration, we first search for the singular points of the integrand where the denominator vanishes, and then apply Cauchy's integral theorem to approximate the complex integral to a summation of the residues and neglect the branch line integral. Note that at the singular points $\left(\eta=\eta_{n}\right)$, the numerator $u_{a}\left(r^{<}, \eta\right) u_{b}\left(r^{>}, \eta\right)$ becomes simply $U_{n}\left(r_{0}, \eta_{n}\right) U_{n}\left(r, \eta_{n}\right)$, where $U_{n}$ is a non-normalized mode determined from the next equation with $\eta_{n}$,

$$
\mathcal{L}\left(\eta_{n}\right) U_{n}=0 ; \quad \mathcal{B}\left(a, \eta_{n}\right) U_{n}=0 \text { and } \mathcal{B}\left(b, \eta_{n}\right) U_{n}=0 .
$$

With these non-normalized modes, the approximated solution of $A$ in Eq. (A9) can be found to be

$$
A(r, \theta) \cong i \sum_{n} \frac{U_{n}\left(r_{0}, \eta_{n}\right) U_{n}\left(r, \eta_{n}\right)}{-\partial C /\left.\partial \eta\right|_{\eta_{n}}} \exp \left(i \eta_{n} \theta\right)
$$

where the denominator can yield the normalization factor $A_{0}$ for $U_{n}$ to reach Eq. (A5) via $G_{n}\left(r, \eta_{n}\right)=A_{0} U_{n}\left(r, \eta_{n}\right)$. Because the variable $C$ is $r_{0} W\left[u_{a}\left(r_{0}, \eta\right), u_{b}\left(r_{0}, \eta\right)\right]=r_{0} u_{a}\left(r_{0}, \eta\right) u_{b}{ }_{b}\left(r_{0}, \eta\right)$ $-r_{0} u_{a}^{\prime}\left(r_{0}, \eta\right) u_{b}\left(r_{0}, \eta\right)$ [see Eqs. (A6) and (A7)], the normalization factor $A_{0}$ can be determined from the next equation,

$$
A_{0}^{-2}=-\left.r_{0} \frac{\partial}{\partial \eta}\left[u_{a}\left(r_{0}, \eta\right) u_{b}^{\prime}\left(r_{0}, \eta\right)-u_{a}^{\prime}\left(r_{0}, \eta\right) u_{b}\left(r_{0}, \eta\right)\right]\right|_{\eta_{n}} .
$$

Following the procedure proposed by Porter (1991), we can in fact calculate the right-hand side of Eq. (A12) using the non-normalized mode $U_{n}$ and the boundary conditions at $r=a$ and $b$. The details are omitted here, and the final result is

$$
\begin{aligned}
A_{0}^{-2}= & 2 \eta_{n} \int_{a}^{b} \frac{U_{n}^{2}\left(r, \eta_{n}\right)}{r} d r-a \frac{\partial f(a, \eta)}{\partial \eta} \frac{\partial(a, \eta)}{g\left(\eta_{n}\right.} U_{n}^{2}\left(a, \eta_{n}\right) \\
& +b \frac{\partial f(b, \eta)}{\partial \eta} \frac{f(b, \eta)}{\eta_{\eta_{n}}} U_{n}^{2}\left(b, \eta_{n}\right) .
\end{aligned}
$$

Abramowitz, M., and Stegun, I. A. (1964). Handbook of Mathematical Functions: With Formulas, Graphs, and Mathematical Tables (National Bureau of Standards Applied Mathematics Series 55, Washington, DC), Chap. 9.

Apel, J., Badiey, M., Chiu, C., Finette, S., Headrick, R., Kemp, J., Lynch, J., Newhall, A., Orr, M., Paseward, B., Tielbrger, D., Turgut, A., von der Heydt, K., and Wolf, S. (1997). "An overview of the 1995 SWARM shallow-water internal wave acoustic scattering experiment," IEEE J. Ocean Eng. 22, 465-500.

Badiey, M., Katsnelson, B. G., Lin, Y.-T., and Lynch, J. F. (2011). "Acoustic multipath arrivals in the horizontal plane due to approaching nonlinear internal waves," J. Acoust. Soc. Am. 129, EL141-EL147.

Badiey, M., Katsnelson, B. G., Lynch, J. F., Pereselkov, S., and Siegmann, W. L. (2005). "Measurement and modeling of three-dimensional sound intensity variations due to shallow-water internal waves," J. Acoust. Soc. Am. 117, 613-625.

Braud, J. P. (1992). "Whispering-gallery mirrors: Fabrication tolerances and the effects of surface imperfections," Appl. Optics 31, 4979-4986.

Buckingham, M. J. (1987). "Theory of three-dimensional acoustic propagation in a wedgelike ocean with a penetrable bottom," J. Acoust. Soc. Am. 82, 198-210.

Carlstone, D. S. (1992). "Radiation damping in the mechanical oscillator," Proc. Okla. Acad. Sci. 72, 45-49.

Dickey, J. W., Frisk, G. V., and Überall, H. (1976). "Whispering Gallery wave modes on elastic cylinders," J. Acoust. Soc. Am. 59, 1339-1346.

Duda, T. F. (2006). "Initial results from a Cartesian three-dimensional parabolic equation acoustical propagation code," Woods Hole Oceanographic Institution Technical Report, WHOI-2006-041, Woods Hole Oceanographic Institution, Woods Hole, MA, http://hdl.handle.net/1912/1428, (Last viewed 11/08/2012). 
Duda, T. F., Lin, Y.-T., and Reeder, D. B. (2011). "Observationally constrained modeling of sound in curved ocean internal waves: Examination of deep ducting and surface ducting at short range," J. Acoust. Soc. Am. 130, $1173-1187$.

Feit, M. D., and Fleck, J. A., Jr. (1978). "Light propagation in graded-index fibers," Appl. Opt. 17, 3990-3998.

Finette, S., and Oba, R. (2003). "Horizontal array beamforming in an azimuthally anisotropic internal wave field," J. Acoust. Soc. Am. 114, 131-144.

Frank, S. D., Badiey, M., Lynch, J. F., and Siegmann, W. L. (2005). "Experimental evidence of three-dimensional acoustic propagation caused by nonlinear internal waves," J. Acoust. Soc. Am. 118, 723-734.

Frisk, G. V. (1994). Ocean and Seabed Acoustics: A Theory of Wave Propagation (Prentice-Hall, Englewood Cliffs, NJ), Chaps. 3 and 4.

Frisk, G. V., Dickey, J. W., and Überall, H. (1975). "Surface wave modes on elastic cylinders," J. Acoust. Soc. Am. 58, 996-1008.

Hardin, R. H., and Tappert, F. D. (1973). "Applications of the split-step Fourier method to the numerical solution of nonlinear and variable coefficient wave equations," SIAM Rev. 15, 423-423.

Hiremath, K. R., Hammer, M., Stoffer, R., Prkna, L. and Čtyroký, J. (2005). "Analytical approach to dielectric optical bent slab waveguides," Opt. Quantum Electron. 37, 37-61.

Jensen, F. B., Kuperman, W. A., Porter, M. B., and Schmidt, H. (1994). Computational Ocean Acoustics (AIP, Melville, NY), Chaps. 5 and 6.

Katsnelson, B. G., and Pereselkov, S. A. (2000). "Low-frequency horizontal acoustic refraction caused by internal wave solitons in a shallow sea," Acoust. Phys. 46, 684-691.

Lin, Y.-T., Duda, T. F., and Lynch, J. F. (2009). "Acoustic mode radiation from the termination of a truncated nonlinear internal gravity wave duct in a shallow ocean area," J. Acoust. Soc. Am. 126, 1752-1765.

Lin, Y.-T., and Lynch, J. F. (2011). "Analytical study of the horizontal ducting of sound by an oceanic front over a slope," J. Acoust. Soc. Am. 131, EL1-EL7.

Lynch, J. F., Colosi, J. A., Gawarkiewicz, G. G., Duda, T. F., Pierce, A. D., Badiey, M., Katsnelson, B. G., Miller, J. E., Siegmann, W., Chiu, C.-S., and Newhall, A. (2006). "Consideration of fine-scale coastal oceanography and 3-D acoustics effects for the ESME sound exposure model," IEEE J. Ocean. Eng. 31, 33-48.

Lynch, J. F., Lin, Y.-T., Duda, T. F., and Newhall, A. E. (2010). "Acoustic ducting, shadowing, refraction and dispersion by curved non-linear internal waves in shallow water," IEEE J. Ocean. Eng. 35, 12-27.
McMahon, K. G., Reilly-Rasaka, L. K., Siegmann, W. L., Lynch, J. F., and Duda, T. F. (2012). "Horizontal Lloyd mirror patterns from straight and curved nonlinear internal waves," J. Acoust. Soc. Am. 131, 1689-1700.

Oba, R., and Finette, S. (2002). "Acoustic propagation through anisotropic internal wave fields: Transmission loss, cross-range coherence, and horizontal refraction," J. Acoust. Soc. Am. 111, 769-784.

Pekeris, C. L. (1948). "Theory of propagation of explosive sound in shallow water," in Propagation of Sound in the Ocean, edited by M. Ewing, J. L. Worzel and C. L. Pekeris, Geol. Soc. Am. Mem. (Geological Society of America, New York), Vol. 27.

Porter, M. B. (1991). "The KRAKEN normal mode program," SACLANTCEN memorandum SM-245 (SACLANT Undersea Research Centre, La Spezia, Italy), Chap. 2.

Preisig, J. C., and Duda, T. F. (1997). "Coupled acoustic mode propagation through continental-shelf internal solitary waves," IEEE J. Ocean. Eng. 22, 256-269.

Rayleigh, L. (1910). "The problem of the whispering gallery," Philos. Mag. 20, 1001-1004.

Righini, G. C., Dumeige, Y., Feron, P., Ferrari, M., Conti, G. N., Ristic, D., and Soria, S. (2011). "Whispering gallery mode microresonators: Fundamentals and application," Riv. Nuovo Cimento 34, $1001-1004$.

Tang, D. J., Moum, J. N., Lynch, J. F., Abbot, P., Chapman, R., Dahl, P. H., Duda, T. F., Gawarkiewicz, G., Glenn, S., Goff, J. A., Graber, H., Kemp, J., Maffei, A., Nash, J. D., and Newhall, A. (2007). "Shallow water '06, A joint acoustic propagation/nonlinear internal wave physics experiment," Oceanogr. 20, 156-167.

Tappert, F. D. (1974). "Parabolic equation method in underwater acoustics," J. Acoust. Soc. Am. 55, S34.

Temme, N. M. (1997). "Numerical algorithms for uniform Airy-type asymptotic expansions," Numer. Algorithms 15, 207-225.

Tindle, C. T., Stamp, A. P., and Guthrie, K. M. (1976). "Virtual modes and the surface boundary condition in underwater acoustics," J. Sound Vib. 49, 231-240.

Williams, A. O., Jr. (1978). "Pseudoresonances and virtual modes in underwater sound propagation," J. Acoust. Soc. Am. 64, 1487-1491.

Zhou, J., Zhang, X., and Rogers, P. H. (1991). "Resonant interaction of sound wave with internal solitons in the coastal zone," J. Acoust. Soc. Am. 90, 2042-2054. 